F. Camilo and B. M. Gaensler, eds.

\title{
The Galactic Population and Birth Rate of Radio Pulsars
}

D. R. Lorimer

\author{
University of Manchester, Jodrell Bank Observatory, UK
}

\begin{abstract}
We review current understanding of the underlying, as opposed to the observed, pulsar population. The observed sample is heavily biased by selection effects, so that surveys see less than $10 \%$ of all potentially observable pulsars. We compare various techniques used to correct the sample for these biases. By far the most significant recent development has been the discovery of over 700 pulsars in the Parkes Multibeam (PM) survey. This new sample is far less affected by selection effects and we use it to make a preliminary analysis of the Galactic pulsar distribution, finding further evidence for a deficit of pulsars in the inner Galaxy.
\end{abstract}

\section{Selection Effects in Pulsar Surveys}

Figure 1 shows the current sample of 1300 pulsars in the ATNF on-line catalog (Hobbs et al., these proceedings) projected onto the Galactic plane. Rather than being distributed about the Galactic center, the majority of pulsars are local objects. Far from being representative of the true population, this sample is heavily biased by a number of selection effects which we now outline below.
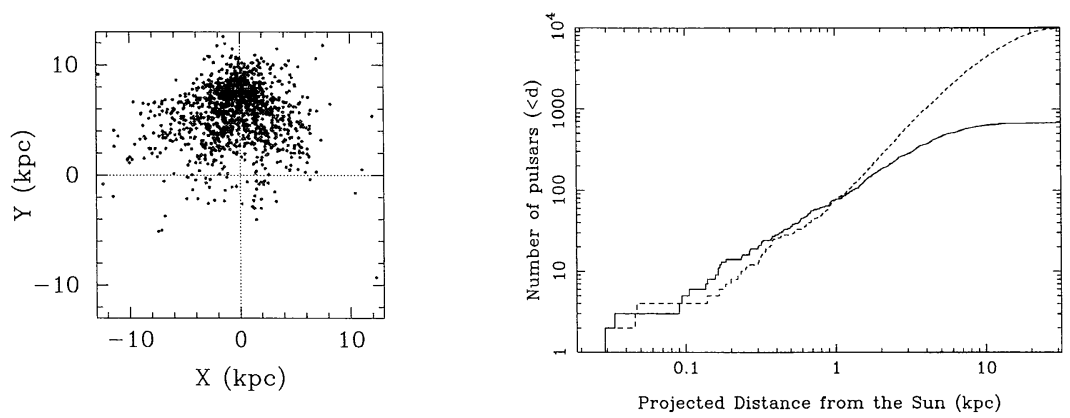

Figure 1. Left: The currently known pulsar population projected onto the Galactic plane. The Galactic center is at the origin and the Sun is at $(0.0,8.5) \mathrm{kpc}$. Right: Cumulative distribution of pulsars as a function of distance from the Sun projected onto the plane. The solid line shows the observed sample (circa 1997) while the dashed line shows the expected distribution of a population free from selection effects. 
The inverse square law. Like all astronomical sources, observed pulsars of a given luminosity $L$ are strongly selected by their apparent flux density, $S$. For pulsars, which beam to a certain fraction $f$ of $4 \pi \mathrm{sr}^{1}, S=L /\left(f 4 \pi d^{2}\right)$, where $d$ is the distance to the pulsar. Since all pulsar surveys have some limiting flux density only those objects bright or close enough will be detectable.

The radio sky background. One limit to pulsar search sensitivities is the thermal noise in the receiver, i.e., the "system temperature", $T_{\text {sys. }}$. While every effort is made to minimize $T_{\text {sys }}$ at the telescope, synchrotron radiating electrons in the Galactic magnetic field contribute significantly with a "sky background" component, $T_{\text {sky }}$. At observing frequencies $\nu \sim 0.4 \mathrm{GHz}, T_{\text {sky }}$ dominates $T_{\text {sys }}$ along the Galactic plane. Fortunately, $T_{\text {sky }} \propto \nu^{-2.8}$ so this is effect is significantly reduced at higher frequencies; e.g., for the PM system, $\nu=1.4 \mathrm{GHz}$.
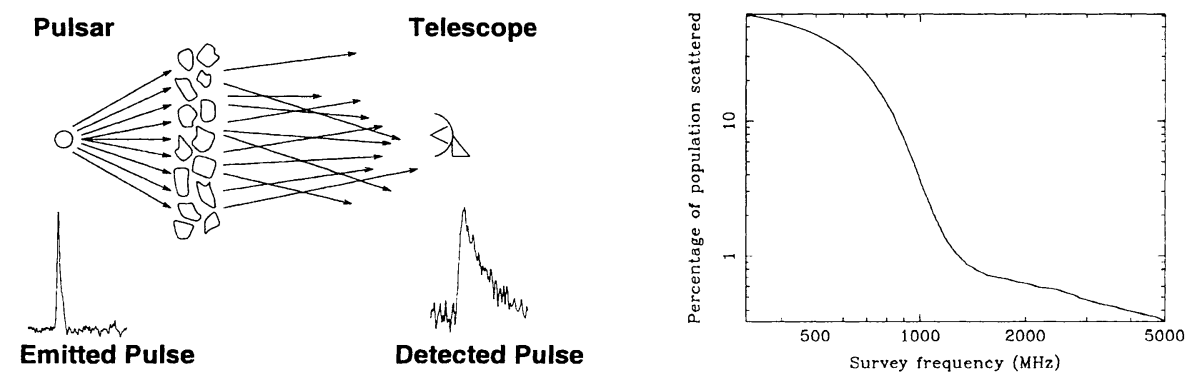

Figure 2. Left: The effect of pulse scattering by irregularities in the ISM. Right: A simulation showing the estimated fraction of pulsars rendered invisible by scattering as a function of observing frequency.

Propagation effects in the interstellar medium (ISM). The ISM is a mixed blessing for pulsar astronomers. On one hand, the dispersion of pulses caused by the ionized component of the ISM permits an estimate of $d$ through the dispersion measure. Conversely, dispersion and scatter-broadening of the pulses conspire against detection of short period and/or distant objects. The effects of scattering are shown in Figure 2. Fortunately, like $T_{\text {sky }}$, the scatterbroadening time $\tau_{\text {scatt }}$ has a strong frequency dependence, scaling roughly as $\nu^{-4}$. As shown in Figure 2, there is a transition frequency of about $1 \mathrm{GHz}$, below which scattering can hide a large fraction of the population. Another factor is scintillation, the modulation of apparent flux densities by refractive or diffractive "screens" of material along the line of sight (Rickett 1970). This is particularly important for nearby pulsars, where the apparent flux densities can vary significantly. For example, two northern sky surveys carried out 20 years apart with comparable sensitivity (Damashek, Taylor \& Hulse 1978; Sayer, Nice $\&$ Taylor 1997) detected a number of pulsars above and below the nominal search thresholds of one experiment but not the other. Ideally, surveying the sky multiple times minimizes the effects of scintillation against pulsars nominally above

\footnotetext{
${ }^{1}$ In the absence of prior knowledge about beaming, geometrical factors are usually ignored and the resulting "pseudoluminosity" is quoted at some standard observing frequency; e.g., at 1400 $\mathrm{MHz}$, given a measured flux density and distance, the pseudoluminosity $L_{1400}=S_{1400} d^{2}$.
} 
the threshold, and maximizes the detections of faint pulsars through favorable scintillation.

Finite size of the emission beam. As mentioned above, the fact that pulsars do not beam to $4 \pi$ sr means that we see only a fraction $f$ of the total active population. For a circular pencil beam, Gunn \& Ostriker (1970) estimated $f \sim 1 / 6$. A consensus on the precise shape and evolution of the emission beam, however, has yet to be reached. Narayan \& Vivekanand (1983) argued that the beam shape is elongated in the meridional direction. Lyne \& Manchester (1988), on the other hand, favor a circular beam. Using the same database, Biggs (1990) presented evidence in favor of meridional compression! All of these studies do agree that the beam size is period dependent, with shorter period pulsars having larger beaming fractions. For example, Tauris \& Manchester (1998) found that $f=0.09[\log (P)-1]^{2}+0.03$, where $P$ is the period in seconds. Undoubtedly, a complete model for $f$ needs to account for other factors, such as evolution of the inclination angle between the spin and magnetic axes. Given the uncertainties, most authors quote results with and without a beaming correction.

Pulse nulling. The abrupt cessation of the pulsed emission for many pulse periods was first identified by Backer (1970). Ritchings (1976) presented evidence that the incidence of nulling became more frequent in older long-period pulsars, suggesting that it signified the onset of the final stages of the neutron star's life as an active radio pulsar. Since most pulsar surveys have short $(<$ few min) integration times, there is an obvious selection effect against nulling objects. Means of combating this effect are to look for individual pulses in search data (see e.g., Nice 1999), survey the sky many times, or use longer integrations. The PM survey, which employs 35-min pointings, is proving particularly effective at detecting nulling pulsars and should soon be able to better quantify this population and provide a more satisfactory understanding of nulling pulsars.

Orbital motion. In standard pulsar searches, where time series are Fourier transformed, the signal from a binary pulsar can be Doppler shifted over several bins in the Fourier domain. In extreme cases, where the survey integration time is a significant fraction of the orbital period, this results in a loss of sensitivity. For example the loss of signal-to-noise of the original binary pulsar B1913+16 during a 35-min observation of the PM survey can be as much as $90 \%$. Correcting for this effect using "acceleration searches" is now becoming more routine, thanks to the ever-increasing availability of high-speed computer resources. Since it is quite possible that young pulsars in tight binary orbits exist, the very deep ( 2-10 hr) searches for young pulsars described by Camilo in this volume are now being re-analyzed with full acceleration searches.

To get an idea of how biased the sample is due to the above effects, Figure 1 shows the cumulative distribution of pulsars as a function of distance from the Sun projected onto the Galactic plane. Also shown is the expected distribution for a simulated population in which there are no selection effects. As can be seen, the two samples are closely matched only out to a $\mathrm{kpc}$ or so before the selection effects become significant. From these curves, we deduce that less than 10\% of the potentially observable population in the Galaxy are currently detectable. Rigorous conclusions about the true pulsar population can only be made after properly accounting for these selection effects. 


\section{Landmark Papers in Pulsar Statistics}

Shortly after the discovery of pulsars, a number of authors began to consider their implications for the Galactic population of neutron stars. A widely cited paper from that era is the work of Gunn \& Ostriker (1970; hereafter GO). To tackle the problem analytically, GO made two simplifying assumptions based on the sample of 52 pulsars known at that time: (a) the relationship $L \propto B^{2}$, where $L$ is the radio luminosity, and $B$ is the dipole magnetic field strength; (b) the evolution of $B$ being an exponential decay with a time constant $t_{d}$. With these in hand, and using the dipolar spin-down expression $B^{2} \propto P \dot{P}$, GO derived expected distributions for the observed population. In particular, they showed that the observed number of pulsars $N_{\text {obs }}=\pi D^{2} F \Sigma t_{d} \exp \left(2 \sigma_{P}^{2}\right) / 2$, where $D$ is the mean distance of the observed sample, $F$ is a parameter relating to the completeness of the surveys, $\Sigma$ is the local birth rate of potentially observable pulsars and $\sigma_{P}$ is derived from their model fit to the observed period distribution. Assuming $f=1 / 6$, and extrapolating the local birth rate over the Galaxy, they arrived at a Galactic birth rate $\mathcal{R}=1 /(30 \mathrm{yr})$, in good agreement with the best estimates of the rate of type-II supernovae at that time (e.g., Blaauw 1961).

Although mathematically appealing, GO's analytical approach required a number of simplifying assumptions about the pulsar population and the Galaxy itself. One of these assumptions, the spontaneous decay of the magnetic field, continues to be extremely controversial. Since GO's original study, many papers have been written presenting arguments for and against field decay (see e.g., Lyne, Manchester \& Taylor 1985; Bailes 1989; Narayan \& Ostriker 1990; Bhattacharya et al. 1992; see also the contribution by van Leeuwen et al. in this volume). A less model-dependent approach to the problem, first developed by Large (1971), can be summarized by the following expression:

$$
d n(P, z, R, L)=V(P, z, R, L) \rho(P, z, R, L) d P d z d R d L .
$$

Here $N$ is the observed population of pulsars as a function of period, $P$, distance from the Galactic plane, $z$, Galactocentric radius, $R$ and luminosity, $L$. The quantity $V$ represents the volume of the Galaxy effectively searched and $\rho$ is the underlying (true) distribution of the population. Since we know $n$ and can estimate $V$ on the basis of (hopefully) well-understood survey sensitivities, we can invert the above expression to solve for $\rho$. The only simplification required to do this is to assume ${ }^{2}$ that $P, z, R$ and $L$ are independent quantities. The problem then reduces to four equations which can be solved for the underlying distributions of interest: $\rho_{P}(P), \rho_{z}(z), \rho_{R}(R)$ and $\rho_{L}(L)$.

Large's method was somewhat ahead of its time: back in 1971, pulsar surveys of the Galaxy were still in their early stages so that $V$ was not well determined. By the late 1970s, however, a number of large-scale searches had been carried out and Taylor \& Manchester (1977; hereafter TM) applied the above technique to the sample of $\sim 150$ pulsars then known (see also Davies, Lyne \& Seiradakis 1977). Integrating the derived distribution functions over the Galaxy,

\footnotetext{
${ }^{2}$ Following a question about this assumption after my talk, I examined the current pulsar sample. Apart from a weak coupling between $P$ and $z$, there are no significant relationships between any of these parameters. The assumption of independent distributions seems to be well founded.
} 
and assuming $f=0.2$, TM estimated the total number of active pulsars in the Galaxy to range between 60000 and 850000 . Here the principle uncertainty is the assumed distance model. TM considered a uniform electron content with a mean electron density in the range $0.02 \mathrm{~cm}^{-3}<n_{e}<0.03 \mathrm{~cm}^{-3}$. To calculate the Galactic birth rate, $\mathcal{R}$, TM required an estimate of the mean pulsar lifetime, $T$, which they obtained from an analysis of the $z$ distribution as a function of characteristic age $\tau=P /(2 \dot{P})$. In the $z-\tau$ diagram (see their Fig. 7), TM argued that the discrepancy between the expected and observed $z$ for characteristic ages larger than a few Myr set a limit to $T=4 \mathrm{Myr}$. This leads to an implied birthrate $\mathcal{R}=1 /(6 \mathrm{yr})$ ! Davies et al. (1977) reached similar conclusions.

The rather high birth rates from these and other analyses prompted Phinney \& Blandford (1981) and in particular Vivekanand \& Narayan (1981; hereafter VN) to consider a less model-dependent approach to estimating $\mathcal{R}$. The method involves binning the observed sample as a function of spin period, $P$, and in each bin computing the flow or "current" of pulsars, $J$, through the bin:

$$
J(P)=\frac{1}{\Delta P} \sum_{i=1}^{n_{\text {bin }}} \frac{\dot{P}_{i} \xi_{i}}{f_{i}} .
$$

Here, $n_{\text {bin }}$ is the number of pulsars in a period bin of width $\Delta P$, while $\xi_{i}$ and $f_{i}$ are the "scale factor" and beaming fraction of the $i^{\text {th }}$ pulsar, respectively. As discussed earlier, $f_{i}$ is based on some beaming model. For a given pulsar, its scale factor $\xi_{i}$ represents the number of pulsars with similar parameters in the Galaxy. In principle, this is very similar to the $V / V_{\max }$ first used to correct observationally biased samples of quasars (Schmidt 1968). In practice $\xi$ is computed using a Monte Carlo simulation of $N$ pulsars with identical periods and luminosities. Using accurate models for the various pulsar surveys, it is relatively straightforward to calculate the number of pulsars $n$ that are detectable from that population. As a result, $\xi=N / n$. Detailed simulations to test this approach show that the scale factors give reliable results about the population of pulsars with luminosities above $L_{\min }$, the minimum luminosity in the sample (see Lorimer et al. 1993). We note in passing that it is possible to form the luminosity function for $L>L_{\min }$ by weighting the observed luminosities by the appropriate scale factors. Integrating this distribution then yields $N\left(>L_{\text {min }}\right)$.

The beauty of the pulsar-current analysis is that it makes just two fundamental assumptions about pulsars: (i) they are a steady-state population; (ii) they are spinning down steadily from short to long periods. The first of these is justified since the ages of pulsars, while not well known $\left(10^{7-8} \mathrm{yr}\right)$, are certainly less than the age of the Galaxy, $10^{10} \mathrm{yr}$. The second is, of course, well in accord with timing observations. The birth-rate can be computed from this analysis by simply plotting $J$ as a function of $P$. In the standard model where pulsars are born spinning rapidly, there should be a peak in the current at short periods followed by a decline in the current as pulsars end their life with longer periods. The birth rate is then just the height of this peak. VN derived $\mathcal{R}=1 /(16-27 \mathrm{yr})$, in much better agreement with the supernova rate.

A by-product of VN's analysis was their conclusion that a significant fraction of pulsars are "injected" into the population with relatively long initial spin periods $\left(P_{0} \sim 0.5 \mathrm{~s}\right)$, rather than the standard picture of birth with $P_{0} \sim 20 \mathrm{~ms}$. Rather like field decay, arguments for and against injection have been presented 
ever since. Following criticism by Lyne, Manchester \& Taylor (1985; hereafter LMT) that VN's analysis had not properly taken account of selection effects such as scattering, Narayan (1987) performed a more detailed analysis which provided further support for injection. Lorimer et al. (1993) used Monte Carlo simulations to investigate the validity of the pulsar current approach and found that it was prone to systematic errors induced by faint, nearby pulsars. Excluding these objects from the analysis significantly reduces the case for injection. Perhaps the final word on this issue will be a detailed pulsar current analysis of the PM survey sample (Vranesevic, these proceedings).

A major effort to quantify the Galactic distribution was the analysis of LMT who combined the theoretical framework of GO with the numerical approach of Large to the sample of 316 pulsars detected in four major $400-\mathrm{MHz}$ surveys. Two key results from their paper are shown in Figure 3. As a result of their numerical analysis, LMT were able to place better constraints on the radial distribution than TM. However, due to the extreme selection effects on $400-\mathrm{MHz}$ surveys towards the inner regions of the Galaxy, the corrected radial distribution becomes very uncertain below $4 \mathrm{kpc}$ (see Fig. 3).
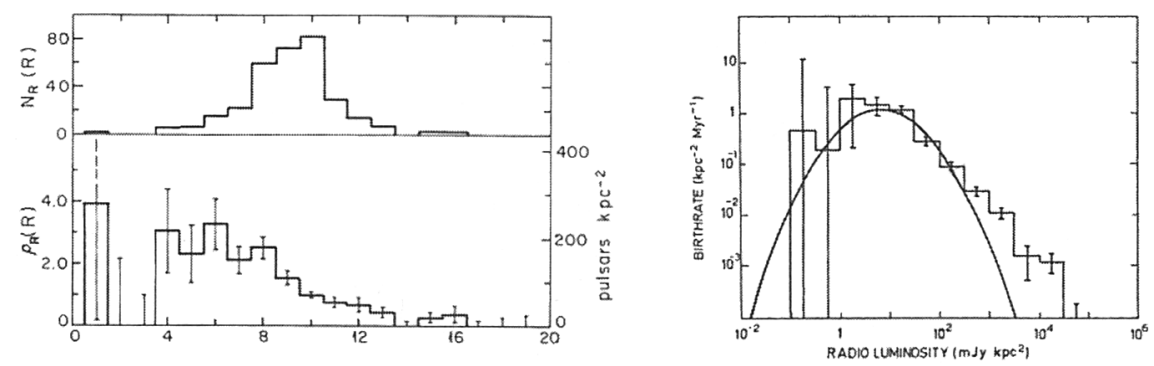

Figure 3. Left: The observed radial distribution (top panel) and corrected radial density function (lower panel) from the numerical analysis of LMT. Right: The birthrate as a function of luminosity assuming the Gunn-Ostriker luminosity model with dipolar spindown and exponential magnetic field decay. The solid line shows the expected distribution of initial luminosities assuming a Gaussian spread in magnetic field.

To derive the birth rate, $\mathcal{R}$, rather than applying a pulsar current analysis, LMT followed GO's approach to model the observed distributions and concluded that the best-fit timescale for exponential field decay to be $9.1 \mathrm{Myr}$. Since their analysis assumed GO's $L \propto B^{2}$ luminosity law, magnetic field decay provided a mechanism for luminosity decay. By considering their corrected luminosity function as a steady-state population where pulsars flowed into successively fainter luminosity bins, LMT were able to derive $\mathcal{R}$ as a function of $L$. The result of this analysis is shown in Figure 3, from which LMT concluded $\mathcal{R}=1 /(30-250 \mathrm{yr})$.

While an elegant approach to the problem, LMTs method relies on the field decay hypothesis being correct. As mentioned previously, the issue of magnetic field decay remains unresolved. Since magnetic field evolution underpins much of pulsar statistics, perhaps the single most important breakthrough in this area would be a comprehensive reassessment of this issue. 


\section{Recent Progress in the Galactic Distribution of Pulsars}

Although much effort has gone into improving the Monte Carlo simulations of pulsar population modeling since LMT, relatively little progress has been made in improving our knowledge of the Galactic distribution. Of particular interest is the underlying density $\rho_{R}$ of pulsars as a function of Galactocentric radius, $R$. LMT and others were only able to place poor constraints on this function (Fig. 3) and most subsequent work has assumed a Gaussian distribution for $\rho_{R}$ (e.g., Narayan 1987). In fact, as pointed out by Bailes \& Kniffen (1992), there is no reason to prefer this function over one where $\rho_{R} \rightarrow 0$ as $R \rightarrow 0$.

Improving our understanding of $\rho_{R}$ at small $R$ requires better statistics of the inner-Galaxy pulsars. Due to the propagation and sky-background selection effects mentioned earlier, low-frequency $(0.4 \mathrm{GHz})$ surveys are very poor probes of this population. One of the main motivations for high-frequency $(1.4 \mathrm{GHz})$ surveys of the Galactic plane is that they are less prone to these effects. Johnston (1994) analyzed two such surveys carried out in the late 1980s (Clifton et al. 1992; Johnston et al. 1992) and found that models with a deficit of pulsars in the inner Galaxy were strongly preferred over a simple Gaussian profile for $\rho_{R}$.

The sample of $\sim 150$ pulsars detected by these two early surveys has now been completely surpassed by the phenomenal success of the PM survey: over 700 new pulsars have been found in the Galactic plane search so far. Together with re-detections of known pulsars, the PM sample amounts to 914 pulsars. I have applied Large's numerical approach to this new sample to derive new distribution functions in $R, L, z$ and $P$. Preliminary results are shown in Figure 4. The corrected radial density function clearly supports Johnston's conclusion for a deficit of pulsars in the inner Galaxy. More work is required in quantifying the significance of this result, particularly in the $R=0.5 \mathrm{kpc}$ bin where only one pulsar is presently known! While a population of inner-Galaxy pulsars could be masked from the PM survey by severe scattering not currently taken into account, it is interesting that the now completed Effelsberg 5-GHz Galactic center survey (Klein et al., these proceedings) has not found a single pulsar.
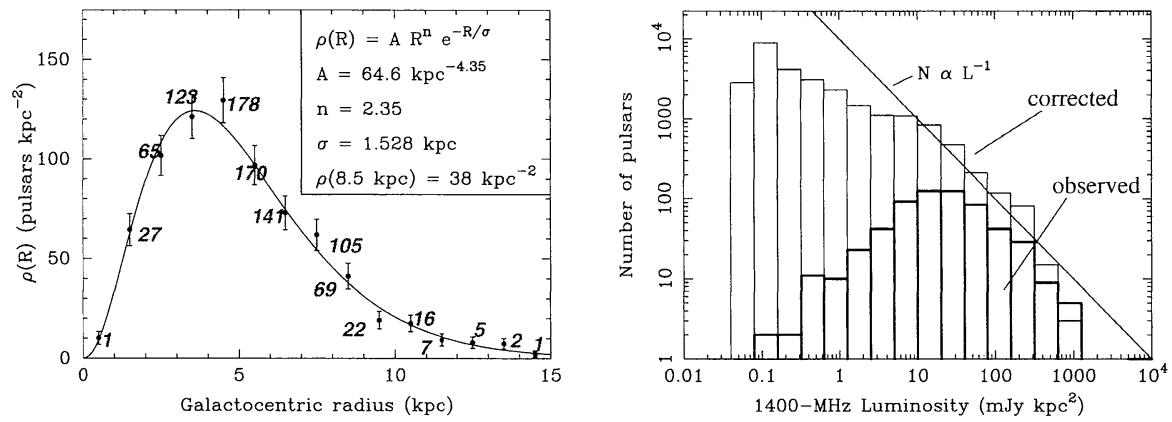

Figure 4. Left: The corrected radial distribution from the PM survey. The inset shows the best-fit analytical function to the data. The number of observed pulsars used to constrain the density in each bin are given. Right: The observed and corrected luminosity distributions. 
Although the PM survey has been the most prolific probe of the Galactic population to date, we are still only scratching the surface. One of many uncertain areas about the pulsar population is the shape of the luminosity function. From Figure 4 we see a clear departure from the $d \log N / d \log L=-1$ relationship at low $L$. Integrating Figure 4 results in a Galactic population of $25000 \pm 2000$ potentially observable pulsars with $L_{1400}>0.3 \mathrm{mJy} \mathrm{kpc}^{2}$. Below this limit, the population is essentially unknown. Future surveys with the Square Kilometer Array, which should easily be able to detect $\sim 15000$ pulsars with $L_{1400}>0.3 \mathrm{mJy} \mathrm{kpc}^{2}$ should also detect many fainter objects and truly constrain the pulsar luminosity function.

Acknowledgments. I thank the Particle Physics and Astronomy Research Council and the Royal Society for supporting my attendance at this meeting.

\section{References}

Backer, D. C., 1970, Nature, 228, 42

Bailes, M., \& Kniffen, D. A. 1992, ApJ, 391, 659

Bailes, M. 1989, ApJ, 342, 917

Bhattacharya, D. et al. 1992, A\&A, 254, 198

Biggs, J. D. 1990, MNRAS, 245, 514

Blaauw, A., 1961, Bull. Astr. Inst. Netherlands, 15, 265

Clifton, T. R. et al. 1992, MNRAS, 254, 177

Damashek, M., Taylor J. H., \& Hulse R. A. 1978, ApJ, 225, L31

Davies, J. G., Lyne, A. G., \& Seiradakis J. H. 1977, MNRAS, 179, 635

Gunn, J. E., \& Ostriker J. P. 1970, ApJ, 160, 979 (GO)

Johnston, S. 1994, MNRAS, 268, 595

Johnston, S. et al. 1992, MNRAS, 255, 401

Large, M. I. 1971, in IAU Symposium No. 46 (Dordrecht: Reidel), p. 165

Lorimer, D. R. et al. 1993, MNRAS, 263, 403

Lyne, A. G., \& Manchester, R. N. 1988, MNRAS, 234, 477

Lyne, A. G., Manchester, R. N., \& Taylor, J. H. 1985, MNRAS, 213, 613 (LMT)

Narayan, R. \& Ostriker, J. P. 1990, ApJ, 352, 222

Narayan, R., \& Vivekanand M. 1983, A\&A, 122, 45

Narayan, R. 1987, ApJ, 319, 162

Nice, D. J. 1999, ApJ, 513, 927

Phinney, E. S., \& Blandford, R. D. 1981, MNRAS, 194, 137

Rickett, B. J. 1970, MNRAS, 150, 67

Ritchings, R. T. 1976, MNRAS, 176, 249

Sayer, R. W., Nice, D. J., \& Taylor, J. H. 1997, ApJ, 474, 426

Schmidt, M. 1968, ApJ, 151, 393

Tauris, T. M., \& Manchester R. N. 1998, MNRAS, 298, 625

Taylor, J. H., \& Manchester R. N. 1977, ApJ, 215, 885 (TM)

Vivekanand M., \& Narayan R. 1981, JA\&A, 2, 315 (VN) 